\title{
Disponibilidad de frutas, hortalizas y legumbres en países de Suramérica en el periodo de 1961 al 2010
}

\section{Availability of fruits, vegetables and legumes in countries the South America during the period 1961 to 2010}

\section{RESUMEN}

La disponibilidad es un factor relevante en la elección y consumo de los alimentos por parte de las poblaciones y se hace relevante cuando se trata de alimentos como las frutas y verduras por su bajo valor energético y alto costo relativo. Objetivo: Efectuar un análisis descriptivo, comparativo referente a la disponibilidad de frutas, verduras y legumbres en los diferentes países de Suramérica en un periodo de cincuenta años. Material y métodos: Estudio analítico retrospectivo, en el que se empleó el Modelo lineal general incorporando la técnica multivariada de la varianza (MANOVA) con contraste canónico de tipo ortogonal, análisis de correlación, estadística descriptiva exploratoria de tipo unidimensional, en una base de datos proveniente de la FAO. Resultados: Brasil, Chile y Colombia incrementaron la disponibilidad de frutas. Venezuela y Uruguay fueron representativos en aumento de hortalizas. Colombia y Uruguay se destacaron por su crecimiento en el suministro de legumbres. Conclusiones: Hubo diferencia entre algunos países de Suramérica. Brasil, Colombia y Ecuador fueron las naciones con mayor per cápita en frutas. Chile, Argentina y Paraguay en hortalizas. En legumbres Brasil fue el que más se destacó. Bolivia ocupó las últimas posiciones en disponibilidad de estás fuentes alimentarias. Palabras clave: Disponibilidad; Frutas; Verduras; Legumbres; Suramérica.

\footnotetext{
ABSTRACT

Availability is a relevant factor in the choice and consumption of food by the population, especially foods of low energy value, such as fruits and vegetables. Objective: To carry out a descriptive and comparative analysis regarding availability of fruits, legumes and vegetables in different South American countries over a 50-year period. Methods and materials: Retrospective analytical study. We used a general linear (MANOVA for multivariate technique of variance) with a canonical contrast of orthogonal type. Using a FAO database, we conducted correlation analysis and exploratory unidimensional type descriptive statistics. Results: Brazil, Chile, and Colombia increased the availability of fruits over the study period. Venezuela and Uruguay were
}

Luz Arboleda', Luís Restrepo², Deisy Pava'.

1. Escuela de Nutrición y Dietética Universidad de Antioquia, Colombia.

2. Facultad de Ciencias Agrarias, Universidad de Antioquia, Colombia.

Dirigir correspondencia a: Luz Arboleda. Escuela de Nutrición y Dietética, Universidad de Antioquia, Colombia. Teléfono 3147728822

E-mail: luzmar.arboleda@gmail.com

Este trabajo fue recibido el 09 de junio de 2017. Aceptado con modificaciones: 24 de octubre de 2017. Aceptado para ser publicado: 03 de enero de 2018.

representative in increase of vegetables. Colombia and Uruguay greatly increased supply oflegumes. Conclusions: There was a significant difference among some countries in South America: Brazil, Colombia and Ecuador were the nations with the highest per capita supply? in fruit. Chile; Argentina and Paraguay had the highest availability for vegetables. Brazil showed the highest rank for legumes. Bolivia had the lowest availability of these 3 food sources. Keywords: Availability; Fruits; Vegetables; Legumes; South America.

\section{INTRODUCCIÓN}

La alimentación siempre ha sido un acto de suma importancia en todos los tiempos, ha permitido el desarrollo 
social y cultural de los diferentes grupos poblacionales y de sociedades enteras. Se constituye en un acto complejo porque en este confluyen variedad de factores como los macrosociales, relacionados con las políticas, la economía y la cultura; y los microsociales, afines a las creencias y simbolismos alimentarios.

Debido a su importancia y complejidad, los gobiernos han instaurado políticas tendientes a mejorar la disponibilidad de alimentos suficientes para toda la población, con el fin de satisfacer los requerimientos nutricionales y alimentarios de los diferentes grupos. La disponibilidad de alimentos es una noción ligada a la seguridad alimentaria y definida como la cantidad de alimentos es definida como la cantidad de alimentos con la que cuenta un país para aportar un suministro suficiente y adecuado de calorías y nutrientes para los grupos poblacionales'. De esta manera, y en concordancia con lo anterior, la disponibilidad de alimentos, es un asunto de suma importancia y relevante para un país.

La disponibilidad de alimentos está determinada por factores como la estructura productiva, los sistemas de comercialización internos y externos, los factores productivos, las condiciones ecosistémicas y las políticas de producción y comercio'.

En el sistema agrícola se cuenta con variedad de grupos de alimentos, clasificados según su procedencia y contenido nutricional, uno de estos grupos es el de hortalizas o verduras, cuyo contenido de calcio, hierro, sobre todo en las hojas verdes ${ }^{2}$, es alto; otro grupo es el de las frutas, con alto contenido en caroteno, vitamina $\mathrm{C}$ y fibra dietética ${ }^{3}$; y el de las legumbres, también muy importantes desde el punto de vista nutricional por su alto contenido de proteínas, y vitaminas del complejo B (excepto B12) ${ }^{4}$. Este último grupo de alimentos se caracteriza porque sus frutos están dispuestos en hileras dentro de vainas (lentejas, arvejas, porotos, garbanzos, soja, maní). Las más consumidas en Suramérica son los fríjoles, arvejas, lentejas, garbanzos y semillas oleaginosas como el ajonjolí y las semillas de girasols.

Los anteriores grupos de alimentos son muy importantes en la alimentación humana, pues debido a su alto contenido de nutrientes, previenen y disminuyen patologías como dislipidemias, hipertensión arterial, obesidad y cáncer colorrectal ${ }^{3,4}$ entre otras.

Desde organismos internacionales como la Organización de las Naciones Unidas para la Alimentación y la Agricultura (FAO), dedicadas a la lucha contra el hambre y a la instauración de la Seguridad Alimentaria y Nutricional en los diferentes países, y la Organización Mundial de la Salud (OMS), se promueve y fomenta el consumo de frutas, verduras y legumbres en las poblaciones ${ }^{6}$, lo cual se hace por considerar que estos alimentos contienen elementos esenciales para llevar una dieta saludable que contribuye a la prevención de enfermedades crónicas como las cardiovasculares, obesidad y algunos cánceres, y evitar así que cada año mueran 1,7 millones?

Lo anterior, ha llevado a la instauración de estrategias, programas y proyectos a nivel internacional, nacional y regional tendientes a aumentar la producción y a la vez a mejorar el consumo de estos alimentos y lograr así que la mayoría de la población pueda alcanzar el consumo recomendado por la Organización Mundial de la Salud (OMS), de cinco porciones de frutas y dos de hortalizas al día ${ }^{8}$.

Dada la importancia de estos alimentos en la prevención de enfermedades crónicas no transmisibles, en la consecución de la seguridad alimentaria y nutricional de los pueblos, y como una manera de fomentar su consumo, el año 2016 fue declarado como el año internacional de las legumbres por la Asamblea General de las Naciones Unidas, con el fin de sensibilizar a la población sobre las ventajas nutricionales de este grupo de alimentos y como estrategia para incentivar su producción de manera sostenible ${ }^{9}$.

Así mismo, desde el año 2004 la Asamblea Mundial de Salud aprobó la Estrategia Mundial sobre Régimen alimentario, Actividad Física y Salud, que propone como una de sus metas y objetivos "aumentar el consumo de frutas, hortalizas y legumbres". Esas directrices orientan la política nacional en materia de alimentación, educación nutricional y otras intervenciones de salud pública ${ }^{10}$.

Respecto a la importancia dada a nivel general al consumo de estos grupos de alimentos, el gobierno de Argentina acordó la elaboración de un Marco Nacional de Prioridades en salud pública, entre las que se destaca la promoción del consumo de frutas y hortalizas ${ }^{11}$.

Los anteriores, son solo una muestra de la importancia que le dan los gobiernos y entidades de salud pública de países de Suramérica, al fomento del consumo y de la adecuada disponibilidad de frutas, hortalizas y legumbres para sus poblaciones. No obstante, y pese a dichos esfuerzos, según estimaciones de la OMS, 2.7 millones de personas mueren anualmente en el mundo, debido a una ingesta insuficiente de frutas y verduras, lo que representa 26,7 millones de pérdida de años de vida.

El consumo actual estimado de frutas y verduras es muy variable en todo el mundo, oscila entre $100 \mathrm{~g} /$ día en los países menos desarrollados y aproximadamente $450 \mathrm{~g} /$ día en Europa Occidental ${ }^{12}$. En Colombia, según la Encuesta Nacional de Situación Nutricional en Colombia 2010 (ENSIN 2010), sólo el 20,5\% de los colombianos consumen frutas tres o más veces cada día y únicamente 3 de cada 10 personas consumen todos los días verduras y hortalizas, de hecho, sólo el 6,4\% lo hacen dos o más veces al día ${ }^{13}$.

Algunas de las causas del bajo consumo de estos alimentos, hacen referencia a la falta de accesibilidad por parte de las personas y familias, a la poca costumbre en su consumo, a su alto costo relativo relacionado con su baja capacidad de saciedad. Además, algunas de las características de su consumo están asociadas con el estrato socioeconómico, siendo las personas de estratos más altos quienes más las consumen y con mayor frecuencia; también están asociadas con el nivel de escolaridad, mientras más alto, su consumo será mayor; y se asocia con la edad y el género, siendo mayor el consumo en población con mayor edad y de género femenino ${ }^{14,15}$. 
Debido a la importancia de estos grupos de alimentos para la salud humana, y para alcanzar la seguridad alimentaria y nutricional de las poblaciones, y convencidos que la disponibilidad y suministro de estos alimentos favorecen su consumo, se realizó este estudio con el objetivo de identificar el suministro de frutas, verduras y legumbres en los países de Suramérica en las últimas cinco décadas.

\section{MATERIALES Y MÉTODOS}

El presente se trata de un estudio analítico retrospectivo, en el cual se llevó a cabo un análisis descriptivo comparativo de tipo multidimensional. Se partió de la información reportada por la FAO, en su portal FAOSTAT, que aportó información referente al suministro de frutas, de hortalizas y de legumbres expresadas en $\mathrm{g} /$ persona/día. Las variables control fueron: país y década, las cuales se ajustaron a una distribución multinomial.

El análisis de la información se realizó por medio del modelo lineal general mediante la técnica multivariada de la varianza MANOVA con contraste canónico ortogonal, estableciendo la dimensionalidad de la comparación por medio del criterio de máxima verosimilitud, observando el mayor valor propio significativo; se aplicó adicionalmente el método de Spearman y el análisis de clúster. Complementándose por medio de estadística descriptiva de tipo unidimensional, para lo cual se utilizaron los paquetes estadísticos SAS University y R versión 3.3.3

\section{RESULTADOS}

Paraguay presentó la mayor disponibilidad en el suministro de frutas en las primeras cuatro décadas evaluadas, cayendo significativamente en el periodo del 2000. Caso contrario al de Colombia, que pasó a ser el país Suramericano con la mayor disponibilidad promedio expresada en g/persona/día $(68,9)$. Bolivia a lo largo del periodo evaluado presentó los valores más bajos en el aporte de frutas para su población. En el caso de Venezuela se observó que el mayor suministro disponible se dio en la década del 60, disminuyendo dicha disponibilidad, en las demás décadas evaluadas. Chile y Brasil han incrementado su aporte en el tiempo (Tabla 1).

En el caso de las hortalizas, Chile presentó diferencia estadística a lo largo de las diferentes décadas evaluadas ( $\mathrm{p}<$ $0,05)$ con los demás países de Suramérica, ostentando el mayor promedio de suministro disponible. Brasil y Colombia ocupan las últimas posiciones en la disponibilidad de hortalizas para su población; y Ecuador, Bolivia, Chile y Argentina han disminuido notoriamente el aporte de este grupo de alimentos a lo largo de las diferentes décadas evaluadas. Caso contrario al de Uruguay y Venezuela, que las han aumentado (Tabla 2).

Brasil es el país de Suramérica con la mayor disponibilidad de suministro de legumbres, presentando diferencia estadística $(p<0,05)$ con respecto a las demás naciones. Por su parte, Argentina, Chile y Ecuador han disminuido su aporte a la población a lo largo de las décadas evaluadas; y Colombia y Uruguay las han aumentado (Tabla 3).

El análisis comparativo entre décadas para cada país de Suramérica, permite destacar los siguientes aspectos en la disponibilidad de suministro de frutas: Bolivia, Ecuador, Venezuela y Paraguay han disminuido el aporte de frutas a la población, caso contrario de Colombia y Chile. En lo referente a la disponibilidad de hortalizas, Brasil, Venezuela y Uruguay presentaron diferencia a favor de la primera década del 2000, lo que indica que hay mayor suministro en el último periodo evaluado; caso contrario a lo ocurrido en Argentina, Colombia, Ecuador y Chile, que han disminuido el suministro de hortalizas a la población. En lo referente a las legumbres, Uruguay fue el único país con incremento significativo $(p<0,05)$ en las dos últimas décadas evaluadas. Ecuador y Chile fueron los países con el mayor retroceso en ese aspecto (Tabla 4).

Tabla 1.

Análisis descriptivo y comparativo por década relacionado con el suministro de frutas en Sudamérica.

\begin{tabular}{lccccc}
\hline País & $\mathbf{6 0}$ & $\mathbf{7 0}$ & $\mathbf{8 0}$ & $\mathbf{9 0}$ & $\mathbf{2 0 0 0}$ \\
\hline Argentina & $37,5 \pm 5,3^{\mathrm{d}}$ & $35,1 \pm 8,2^{\mathrm{c}}$ & $29,4 \pm 4,0^{\mathrm{cd}}$ & $39,6 \pm 7,0^{\mathrm{c}}$ & $34,5 \pm 4,7^{\mathrm{d}}$ \\
Bolivia & $25,4 \pm 0,5^{\mathrm{e}}$ & $26,1 \pm 1,5^{\mathrm{d}}$ & $27,0 \pm 1,8^{\mathrm{d}}$ & $29,4 \pm 0,7^{\mathrm{d}}$ & $20,5 \pm 7,2^{\mathrm{e}}$ \\
Brasil & $38,4 \pm 1,7^{\mathrm{d}}$ & $38,7 \pm 3,5^{\mathrm{c}}$ & $40,7 \pm 5,6^{\mathrm{b}}$ & $51,4 \pm 3,9^{\mathrm{c}}$ & $61,9 \pm 5,1^{\text {ab }}$ \\
Chile & $34,0 \pm 2,1^{\mathrm{d}}$ & $38,4 \pm 5,7^{\mathrm{c}}$ & $34,8 \pm 6,8^{\mathrm{cb}}$ & $49,6 \pm 9,2^{\mathrm{ab}}$ & $54,3 \pm 7,6^{\mathrm{b}}$ \\
Colombia & $13,0 \pm 0,0^{\mathrm{f}}$ & $13,2 \pm, 0,6^{\mathrm{e}}$ & $16,9 \pm 7,9^{\mathrm{e}}$ & $46,9 \pm 8,5^{\mathrm{ab}}$ & $68,9 \pm 5,9^{\mathrm{a}}$ \\
Ecuador & $74,0 \pm 8,6^{\mathrm{a}}$ & $51,1 \pm 5,2^{\mathrm{b}}$ & $40,2 \pm 4,7^{\mathrm{b}}$ & $43,1 \pm 28,5^{\mathrm{bc}}$ & $65,3 \pm 9,4^{\mathrm{a}}$ \\
Paraguay & $75,4 \pm 3,5^{\mathrm{a}}$ & $66,6 \pm 4,0^{\mathrm{a}}$ & $62,0 \pm 4,5^{\mathrm{a}}$ & $48,6 \pm 5,2^{\text {ab }}$ & $40,2 \pm 1,4^{\text {cd }}$ \\
Perú & $51,7 \pm 4,8^{\mathrm{c}}$ & $63,9 \pm 7,3^{\mathrm{a}}$ & $36,9 \pm 1,5^{\mathrm{cb}}$ & $40,7 \pm 5,9^{\mathrm{bc}}$ & $53,9 \pm 3,6^{\mathrm{b}}$ \\
Uruguay & $32,6 \pm 4,9^{\mathrm{d}}$ & $33,1 \pm 10,4^{\mathrm{cd}}$ & $25,2 \pm 7,1^{\mathrm{d}}$ & $46,3 \pm 9,3^{\mathrm{ab}}$ & $43,1 \pm 6,9^{\mathrm{c}}$ \\
Venezuela & $58,6 \pm 5,3^{\mathrm{b}}$ & $49,2 \pm 3,8^{\mathrm{b}}$ & $38,2 \pm 5,3^{\mathrm{b}}$ & $34,7 \pm 3,2^{\mathrm{c}}$ & $44,6 \pm 4,6^{\mathrm{c}}$
\end{tabular}

Elaboración propia con base en la información reportada por la FAO. Los valores son mostrados como media \pm DE. Letras distintas indican diferencia estadística significativa $(p<0.05)$, con base en la prueba de Tukey. 
Tabla 2.

Análisis descriptivo y comparativo por década relacionado con el suministro de hortalizas en Sudamérica.

\begin{tabular}{|c|c|c|c|c|c|}
\hline País & 60 & 70 & 80 & 90 & 2000 \\
\hline Argentina & $163,4 \pm 4,5^{\mathrm{b}}$ & $140,1 \pm 14,3^{b}$ & $124,6 \pm 5,5^{b}$ & $118,4 \pm 3,7^{b}$ & $107,1 \pm 2,6^{b}$ \\
\hline Bolivia & $122,2 \pm 12,5^{d}$ & $125,9 \pm 8,7^{b}$ & $113,3 \pm 17,7^{b}$ & $110,0 \pm 3,9^{\mathrm{bc}}$ & $80,4 \pm 22,1^{\mathrm{d}}$ \\
\hline Brasil & $43,5 \pm 2,4^{h}$ & $39,7 \pm 1,4^{f}$ & $39,7 \pm 1,6^{\text {de }}$ & $44,8 \pm 3,7^{\text {ef }}$ & $59,7 \pm 7,9^{\mathrm{e}}$ \\
\hline Chile & $232,1 \pm 8,9^{a}$ & $205,1 \pm 15,0^{a}$ & $191,4 \pm 34,1^{a}$ & $180,2 \pm 15,5^{a}$ & $150,4 \pm 9,6^{a}$ \\
\hline Colombia & $49,7 \pm 6,2^{\mathrm{hg}}$ & $66,0 \pm 8,8^{\mathrm{e}}$ & $58,6 \pm 12,7^{\mathrm{cd}}$ & $31,9 \pm 2,9^{f}$ & $46,3 \pm 3,8^{e}$ \\
\hline Ecuador & $142,2 \pm 25,9^{c}$ & $77,2 \pm 16,5^{\mathrm{de}}$ & $64,7 \pm 6,1^{c}$ & $54,2 \pm 6,7^{e}$ & $47,5 \pm 6,2^{\mathrm{e}}$ \\
\hline Paraguay & $105,3 \pm 4,4^{\mathrm{e}}$ & $104,5 \pm 6,3^{c}$ & $125,3 \pm 14$, bo & $100,5 \pm 4,3^{c}$ & $103,6 \pm 9,9^{b c}$ \\
\hline Perú & $87,6 \pm 8,7^{f}$ & $85,3 \pm 8,8^{\mathrm{d}}$ & $54,3 \pm 3,0^{\text {cd }}$ & $60,7 \pm 11,9^{\mathrm{e}}$ & $91,2 \pm 15,6^{\text {cd }}$ \\
\hline Uruguay & $60,8 \pm 6,8^{g}$ & $63,2 \pm 0,4^{\mathrm{e}}$ & $64,3 \pm 1,5^{c}$ & $80,4 \pm 17,5^{d}$ & $93,5 \pm 5,6^{\text {cd }}$ \\
\hline Venezuela & $22,2 \pm 0,4^{i}$ & $26,3 \pm 3,5^{f}$ & $27,3 \pm 2,6^{e}$ & $60,4 \pm 23,2^{\mathrm{e}}$ & $87,7 \pm 8,9^{d}$ \\
\hline
\end{tabular}

Tabla 3.

Análisis descriptivo y comparativo por década relacionado con el suministro de legumbres en Sudamérica.

\begin{tabular}{|lccccc|}
\hline Década & 60 & 70 & 80 & 90 & 2000 \\
\hline Argentina & $5,7 \pm 1,3^{\mathrm{f}}$ & $3,1 \pm 0,6^{\mathrm{e}}$ & $3,7 \pm 0,9^{\mathrm{f}}$ & $3,5 \pm 0,5^{\mathrm{f}}$ & $3,0 \pm 1,5^{\mathrm{g}}$ \\
Bolivia & $6,3 \pm 0,7^{\mathrm{f}}$ & $7,0 \pm 1,4^{\text {de }}$ & $9,6 \pm 1,6^{\mathrm{ed}}$ & $7,0 \pm 1,5^{\mathrm{ef}}$ & $6,0 \pm 1,2^{\mathrm{fg}}$ \\
Brasil & $63,8 \pm 5,7^{\mathrm{a}}$ & $54,1 \pm 10,1^{\mathrm{a}}$ & $43,0 \pm 2,6^{\mathrm{a}}$ & $43,8 \pm 4,7^{\mathrm{a}}$ & $45,2 \pm 1,7^{\mathrm{a}}$ \\
Chile & $16,6 \pm 1,7^{\mathrm{d}}$ & $16,6 \pm 2,2^{\mathrm{c}}$ & $13,2 \pm 4,2^{\text {cd }}$ & $9,2 \pm 1,1^{\text {de }}$ & $11,2 \pm 1,5^{\mathrm{e}}$ \\
Colombia & $12,6 \pm 1,0^{\mathrm{e}}$ & $12,7 \pm 0,8^{\mathrm{cd}}$ & $16,2 \pm 1,0^{\mathrm{cb}}$ & $19,1 \pm 1,0^{\mathrm{c}}$ & $19,3 \pm 1,2^{\mathrm{c}}$ \\
Ecuador & $32,1 \pm 3,3^{\mathrm{b}}$ & $18,6 \pm 5,9^{\mathrm{c}}$ & $10,5 \pm 2,2^{\mathrm{d}}$ & $11,7 \pm 1,5^{\mathrm{d}}$ & $7,2 \pm 1,4^{\mathrm{f}}$ \\
Paraguay & $26,3 \pm 2,3^{\mathrm{c}}$ & $45,7 \pm 10,4^{\mathrm{b}}$ & $40,8 \pm 6,9^{\mathrm{a}}$ & $29,0 \pm 4,5^{\mathrm{b}}$ & $29,5 \pm 5,7^{\mathrm{b}}$ \\
Perú & $24,2 \pm 2,5^{\mathrm{c}}$ & $17,0 \pm 2,4^{\mathrm{c}}$ & $17,6 \pm 1,8^{\mathrm{cb}}$ & $16,6 \pm 3,5^{\mathrm{c}}$ & $19,6 \pm 1,7^{\mathrm{c}}$ \\
Uruguay & $4,7 \pm 0,5^{\mathrm{f}}$ & $5,0 \pm 0,7^{\mathrm{e}}$ & $5,4 \pm 0,7 \mathrm{ef}$ & $7,3 \pm 0,8^{\mathrm{e}}$ & $8,3 \pm 1,0^{\text {ef }}$ \\
Venezuela & $23,0 \pm 1,9^{\mathrm{c}}$ & $18,2 \pm 2,9^{\mathrm{c}}$ & $18,4 \pm 3,8^{\mathrm{b}}$ & $15,6 \pm 2,2^{\mathrm{c}}$ & $15,9 \pm 3,2^{\mathrm{d}}$ \\
Elaboración propia con base en la información reportada por la FAO. Los valores son mostrados como media \pm DE. Letras distintas indican diferencia \\
estadística significativa $(\mathrm{p}<0.05)$, con base en la prueba de Tukey. & & & \\
\hline
\end{tabular}

Tabla 4.

Análisis comparativo entre décadas por país, para el suministro de frutas, hortalizas y legumbres.

\begin{tabular}{|c|c|c|c|c|c|c|c|c|c|c|c|c|c|c|c|}
\hline & \multicolumn{5}{|c|}{ Frutas } & \multicolumn{5}{|c|}{ Hortalizas } & \multicolumn{5}{|c|}{ Legumbres } \\
\hline & 60 & 70 & 80 & 90 & 2000 & 60 & 70 & 80 & 90 & 2000 & 60 & 70 & 80 & 90 & 2000 \\
\hline Ar & 14,3 & 23,5 & 13,7 & 17,5 & 13,7 & 2,9 & 10,3 & 4,4 & 3,1 & 2,5 & 22,5 & 18,3 & 25,6 & 15,1 & 49,5 \\
\hline $\mathrm{Br}$ & 4,5 & 8,9 & 13,8 & 7,7 & 8,3 & 5,5 & 3,6 & 3,9 & 8,3 & 13,2 & 8,9 & 18,7 & 6,0 & 10,7 & 3,7 \\
\hline $\mathrm{Ch}$ & 6,1 & 14,8 & 19,6 & 18,6 & 13,9 & 3,8 & 7,3 & 17,8 & 8,6 & 6,4 & 10,4 & 13,4 & 31,5 & 12,4 & 13,2 \\
\hline Co & 0,0 & 4,8 & 47,3 & 18,2 & 8,5 & 12,5 & 13,4 & 21,7 & 9,1 & 8,3 & 8,1 & 6,5 & 6,4 & 5,2 & 6,2 \\
\hline $\mathbf{P a}$ & 4,7 & 6,1 & 7,2 & 10,7 & 3,5 & 4,2 & 6,0 & 11,2 & 4,2 & 9,6 & 8,9 & 22,8 & 16,9 & 15,5 & 19,2 \\
\hline Pe & 9,3 & 11,4 & 3,9 & 14,6 & 6,8 & 9,9 & 10,4 & 5,5 & 19,7 & 17,1 & 10,5 & 14,1 & 10,1 & 21,1 & 8,6 \\
\hline Ur & 15,0 & 31,4 & 28,0 & 19,9 & 16,0 & 11,2 & 0,7 & 2,3 & 21,7 & 6,0 & 10,7 & 13,3 & 12,9 & 11,3 & 12,2 \\
\hline Ve & 9,1 & 7,7 & 14,0 & 9,3 & 10,4 & 2,0 & 13,4 & 9,5 & 38,6 & 10,2 & 8,1 & 16,1 & 20,9 & 13,9 & 20,2 \\
\hline
\end{tabular}


Al efectuar el análisis multivariado de la varianza con base en la disponibilidad de frutas, hortalizas y legumbres de manera simultánea, se detectó diferencia altamente significativa $(p<0,0001)$ entre los países evaluados para el periodo de cincuenta años. El análisis canónico permitió evidenciar diferencia a favor de Chile, como el país con mayor suministro conjunto de frutas, hortalizas y legumbres para su población. Colombia, Uruguay y Venezuela fueron los países con el menor suministro conjunto a lo largo del tiempo (Tabla 5).

\section{DISCUSIÓN}

El suministro de alimentos es un factor que influye de manera crucial en el acceso y consumo de la alimentación, pues la mayor disponibilidad hace que estos productos se adquieran a menor costo, y sea de más fácil adquisición para la población.

En las sociedades actuales, donde los individuos tienen el tiempo limitado, la disponibilidad y cercanía para acceder a los alimentos son factores que condicionan la compra y consumo ${ }^{16,17}$; lo cual se puede corroborar estableciendo un análisis de la relación del suministro, con el consumo de los grupos de alimentos de frutas, verduras y legumbres en los diferentes países de Suramérica. En Colombia por ejemplo, según la ENSIN 2010 el 69,5\% de la población consume frutas y tan sólo un 29,9\% hortalizas o verduras, y de acuerdo a los resultados de esta investigación, el suministro de frutas es mayor que el de verduras en este país de Suramérica ${ }^{13}$.
En Chile, a diferencia de Colombia, el mayor consumo reportado por la Encuesta Nacional de Consumo, se da en el grupo de verduras mostrando una prevalencia de $99,3 \%$, seguido de las frutas y de las leguminosas, que se establece en un $95 \%$ y $82 \%$ respectivamente, en todos los grupos de edad, área de residencia, macro-zona y nivel socioeconómico ${ }^{18}$; de acuerdo con los resultados de esta investigación, este es el país que tiene mayor suministro conjunto de verduras, frutas y legumbres.

De acuerdo con este estudio, Argentina fue el país que mayor suministro del grupo de legumbres presentó en la última década en Sudamérica, y a pesar de tan alto suministro, el consumo "de las legumbres es muy bajo" según la Dirección de Industria Alimentaria y Agroindustrias ${ }^{19}$, entidad que reporta 700 gramos per cápita, en comparación con los consumos anuales per cápita de Perú y Chile, que corresponden a $2.500 \mathrm{gr}^{20}$ y $1.100 \mathrm{gr}$ respectivamente ${ }^{21}$.

En este caso se podría pensar que, entonces, la disponibilidad no influye en el consumo de alimentos; pero este caso es especial, pues Argentina es el país que más legumbres exporta a nivel mundial, lo cual corresponde al $80 \%{ }^{19}$. De esta manera, las altas exportaciones de alimentos, sin considerar los requerimientos a nivel nacional, se constituye en un factor que contribuye con la disminución en el consumo de alimentos, porque estos están menos disponibles para la población.

Así como la disponibilidad incide en el acceso; el consumo que se tenga de los alimentos, es un factor que incide en la formación del gusto y de los hábitos alimentarios

Tabla 5.

Análisis multivariado de la varianza por década, complementado con el contraste canónico.

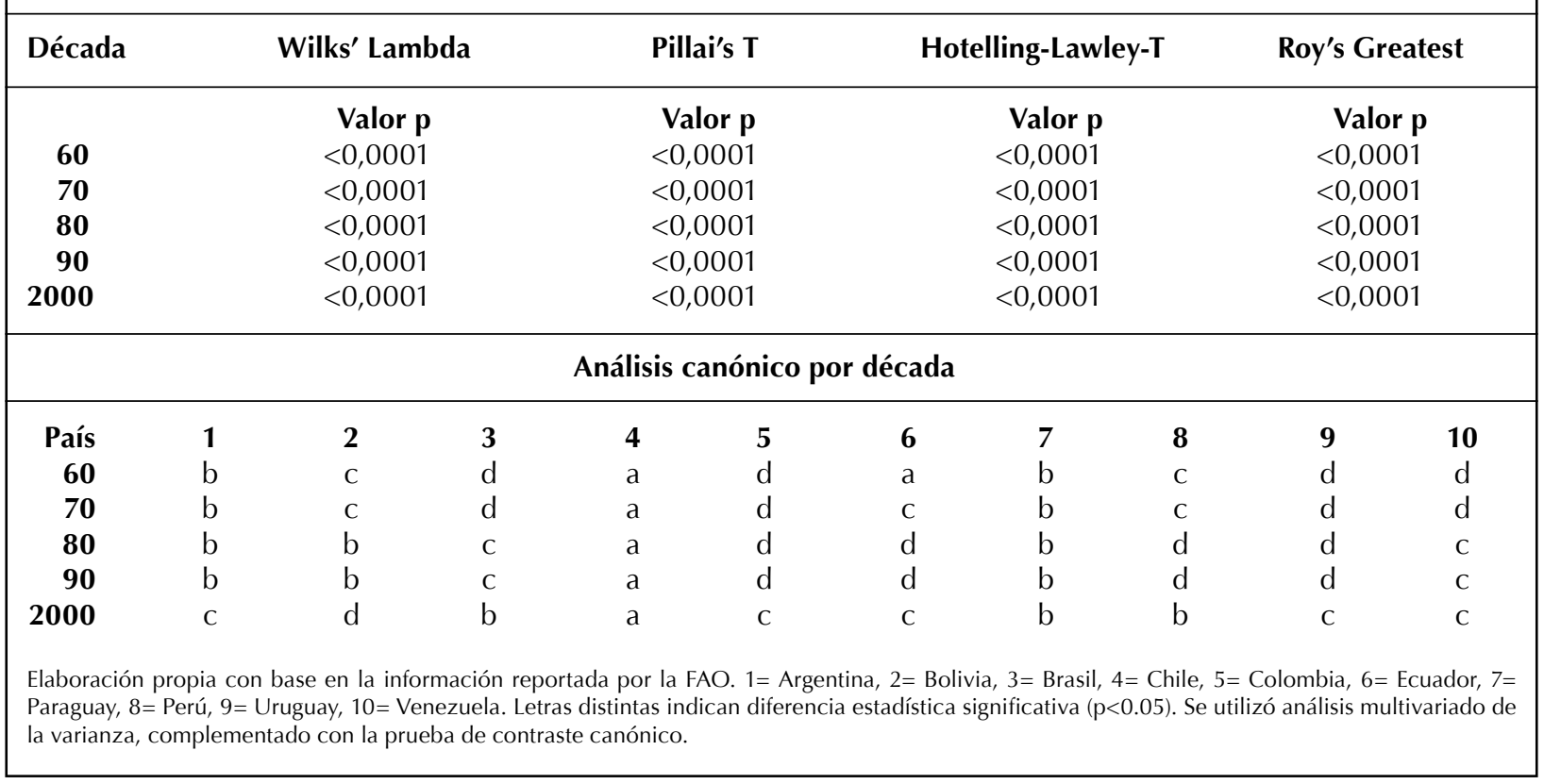


de la población, pues el consumo prolongado hace que se tenga mayor familiaridad con los alimentos y, como afirma Fischler ${ }^{22}$, este es un factor de gran influencia en la formación del gusto, siendo esta categoría, gusto, de gran relevancia en la selección de los alimentos. Es decir, que en el consumo de alimentos, se instaura un círculo, donde los alimentos de mayor disponibilidad son los de mayor acceso, lo que conlleva a que sean de mayor preferencia $y$, por consiguiente, de mayor consumo.

Además de los anteriores factores, en la elección y el consumo de alimentos también influyen factores económicos, los cuales son relevantes y cobran importancia en el momento en que los grupos más empobrecidos de la sociedad, quieren acceder a los alimentos; para estos la relación costo-saciedad de los alimentos es un criterio de elección.

Dicho aspecto, es decir, que un alimento produzca saciedad, no favorece la elección de frutas y verduras, pues su aporte calórico y de saciedad son bajos; y con el dinero que adquieren estos alimentos, también podrían comprar otros como los tubérculos, cereales y grasas que tienen mayor poder de saciedad; por eso son los alimentos más característicos en los hogares más pobres ${ }^{14}$ y es corroborado en numerosos estudios que concluyen que los grupos poblacionales más pobres tienen un consumo elevado de cereales, tubérculos, plátanos y grasas ${ }^{14,17}$.

Lo anterior va en contravía con las recomendaciones dadas en el campo nutricional y de la salud pública, pero no porque los grupos poblacionales no quieran acatar dichas recomendaciones, ni porque no se interesen por su salud; si no porque sus condiciones sociales y económicos les impiden acceder a una dieta adecuada nutricionalmente y saludable, por eso, mantener un suministro adecuado y suficiente de los diferentes grupos de alimentos, sobretodo de los más saludables, es imperativo en los diferentes países.

\section{CONCLUSIONES}

La disponibilidad frecuente y estable del grupo de alimentos de frutas, hortalizas y legumbres se constituye en un factor preponderante que influye en el aumento del consumo de estos alimentos y por consiguiente, en la protección de enfermedades crónicas, degenerativas y cardiovasculares de los grupos poblacionales.

La disponibilidad de frutas entre las décadas de los sesenta y la primera del 2000 ha sido diferente al interior y entre los países de Suramérica; mientras Paraguay presentó una gran disponibilidad de este grupo de alimentos en las primeras cuatro décadas del estudio, Venezuela lo hizo en la primera década, Chile y Brasil fueron aumentando la disponibilidad década a década y Colombia pasó a tener la mayor disponibilidad de este grupo de alimentos.

Los países también presentaron diferencias en las disponibilidades de hortalizas, en este caso fue Chile el que presentó la mayor disponibilidad durante todo el tiempo, Uruguay y Venezuela la aumentó; Ecuador, Bolivia, Chile y Argentina la disminuyeron y Brasil y Colombia presentaron las disponibilidades más bajas de este grupo de alimentos. Lo propio sucedió para las legumbres, pero fue Brasil el que presentó la mayor disponibilidad; Colombia y Uruguay la aumentaron y Argentina, Chile y Ecuador la disminuyeron.

Respecto a la disponibilidad de los tres grupos de alimentos en forma conjunta en los países, Chile fue el que presentó la mayor y Colombia, Uruguay y Venezuela, la menor disponibilidad.

\section{BIBLIOGRAFÍA}

1. Ministry of Social Protection, Ministry of Agriculture and Rural Development, Ministry of National Education, Colombian Family Welfare Institute, Colombian Rural Development Institute. Social Conpes Document 113. National Politic on Food and Nutrition Security (PSAN). Bogotá 2007. [Consulted 2016 November 22]; http://www.icbf.gov.co/portal/page/portal/ Portal/CBF/bienestar/nutricion/pnsan/CONPES113de2008. pdf

2. School of Basic Sciences Technology and Engineering. Fruit and Vegetable Technology. [Consulted 2016 November 22]. http://datateca.unad.edu.co/contenidos/301107/301107II-2013/Act.3_Reconocimiento_unidad_1.pdf

3. Department of Agriculture. FAO Document Depot: Human Nutrition in the Developing World, Chapter 28 Vegetables and Fruits. 2002. [Consulted 2016 December 14] http://www. fao.org/docrep/006/w0073s/w0073s0w.htm\#TopOfPage

4. Sánchez $R$, Fuentes MM, Palma S, López B, Bermejo LM, Gómez C. Fiber-type indication among different pathologies. Nutr Hosp 2015; 31(6): 2372-2383.

5. FAO, Department of Agriculture. Document Depot: Human Nutrition in the Developing World, Chapter 27 Pulses, Nuts and Oilseeds. [Consulted 2016 December 14] http://www. fao.org/docrep/006/w0073s/w0073s0v.htm\#TopOfPage

6. FAO delivers experiences to promote consumption of fruits and vegetables in the population. Food and Agriculture Organization of the United Nations. FAO Regional Office for Latin America and the Caribbean. Santiago, Chile. 2013. [Consulted 2016 December 14]. http://www.fao.org/americas/ noticias/ver/es/c/230290/

7. World Health Organization. Promoting global consumption of fruits and vegetables. [Consulted 2016 December 3]. http:// www.who.int/dietphysicalactivity/fruit/es/

8. Healthy food. Data and numbers. World Health Organization. [Consulted 2016 December 3]. http://www.who.int/mediacentre/ factsheets/fs394/es/

9. Food and Agriculture Organization of the United Nations (FAO). About the International Year of Pulses. Nutritious seeds for a sustainable future. Roma, Italia. 2016. [consulted 2016 November 22]. http://www.fao.org/pulses-2016/about/es/

10. World Health Organization. Global Strategy on Diet, Physical Activity and Health. 2004. [consulted 2016 November 28]. http://www.who.int/dietphysicalactivity/strategy/ eb11344/ strategy_spanish_web.pdf

11. Food and Agriculture Organization of the United Nations. Programs in Argentina. Argentina, 2014. [Consulted 2016 November 28]. http://www.fao.org/argentina/ programasy-proyectos/programa/en/

12. Ministry of Health and Social Protection. Food Guide Document Healthy. Santiago de Cali, 2013. [Consulted 2016 December 3]. https://www.minsalud.gov.co/sites/rid/Lists/BibliotecaDigital/ RIDE/VS/PP/SNA/Guia-Alimentacion-saludable.pdf

13. National Survey of Nutrition Situation in Colombia 2010 ENSIN. 
Profamilia, National Institute of Health, Family Welfare. 2010. [Internet] [Consulted 2016 December 3] Available in: http:// www.icbf.gov.co/portal/page/portal/Descargas1/Resumenfi. pdf

14. Arboleda LM, Duque MM, Urrea JA. Meanings of fruit and vegetable consumption in two rural communities in the municipality of Turbo, Urabá Antioqueño. Saude Soc 2013; 22(4): 1247-1256.

15. Cámara M, Sánchez MC, Torija ME. Nutrition and Health, fruits and vegetables, health sources. Alcobendas, Madrid. 2008. [Consulted 2016 December 2]; http://www.madrid. org/cs/Satellite?blobcol=urldata\&blobheader=application $\% 2 F p d f \&$ blobheadername $1=$ Content-Disposition\&blobhea dervalue1=filename\%3DT034.pdf\& blobkey=id\&blobtable $=$ MungoBlobs\&blobwhere $=1352883450648 \&$ ssbinary $=$ true

16. Matthew, J. Fruits and vegetables are beneficial to health. What are the evidences? [Consulted 2016 December 2]; http://www.frutas-hortalizas.com/articulos/fh_beneficiosas. $p d f$
17. Aguirre P. Rich poor and fat poor. Food crisis. Keys to all. Buenos Aires: Capital intelectual. (2004a).

18. University of Chile. National Food Consumption Survey 20102011. [Consulted 2016 November 20]; http://web.minsal.cl/ sites/default/files/ENCA-INFORME_FINAL.pdf

19. Lezcano E. Argentinian food dried legumes. [Consulted 2016 December 2]; http://www.alimentosargentinos.gov.ar/Contenido/ revista/ediciones/45/cadenas/r45_08_LegumbresSecas.pdf

20. Management The newspaper of economy and business of Peru. Vegetable consumption will double by the end of 2016 the current 2.5 kilos in Peru. 2015. [Consulted 2016 November 28]. http://gestion.pe/economia/consumo-legumbres-duplicarafines-2016-25-kilos-actuales-2143506

21. Iriarte L. Economy and business. Chileans almost eliminate legumes and eat 230 grams of meat a day. 2012. [Consulted 2016 November 28]. http://www.economiaynegocios.cl/ noticias/noticias.asp?id=100702

22. Fischler C. EI (H) Omnivore: The taste, the kitchen and the body. Barcelona. Anagrama 1995. 